\title{
Repeat Rifaximin for Irritable Bowel Syndrome: No Clinically Significant Changes in Stool Microbial Antibiotic Sensitivity
}

\author{
M. Pimentel ${ }^{1}$ - B. D. Cash ${ }^{2}$ - A. Lembo Le R. A. Wolf $^{4} \cdot$ R. J. Israel ${ }^{4}$. \\ P. Schoenfeld ${ }^{5}$
}

Received: 2 December 2016/Accepted: 29 April 2017/Published online: 6 June 2017

(C) Springer Science+Business Media New York 2017

\begin{abstract}
Background Rifaximin has demonstrated efficacy and safety for diarrhea-predominant irritable bowel syndrome (IBS-D).

Aim To determine the rifaximin repeat treatment effect on fecal bacterial antibiotic susceptibility.

Methods Patients with IBS in Trial 3 (TARGET 3) study who responded to open-label rifaximin $550 \mathrm{mg}$ three times daily for 2 weeks, with symptom recurrence within 18 weeks, were randomized to double-blind treatment: two 2-week repeat courses of rifaximin or placebo, separated by
\end{abstract}

R. A. Wolf: Former employee of Salix Pharmaceuticals, 400 Somerset Corporate Blvd., Bridgewater, NJ 08807, USA.

Guarantor of the article: Mark Pimentel.

Electronic supplementary material The online version of this article (doi:10.1007/s10620-017-4598-7) contains supplementary material, which is available to authorized users.

M. Pimentel

Mark.Pimentel@cshs.org

1 GI Motility Program, Division of Gastroenterology, CedarsSinai Medical Center, 8730 Alden Drive, Suite 225E, Los Angeles, CA 90048, USA

2 Division of Gastroenterology, University of South Alabama, 6000 University Commons, 75 University Blvd, S, Mobile, AL 36688, USA

3 Beth Israel Deaconess Medical Center, 330 Brookline Avenue, Boston, MA 02215, USA

4400 Somerset Corporate Blvd., Bridgewater, NJ 08807, USA

5 Gastroenterology and Hepatology Division, John D. Dingell VA Medical Center, 4646 John R Street, Detroit, MI 48201, USA
10 weeks. Prospective stool sample collection occurred before and after open-label rifaximin, before and after the first repeat course, and at the end of the study. Susceptibility testing was performed with 11 antibiotics, including rifaximin and rifampin, using broth microdilution or agar dilution methods.

Results Of 103 patients receiving open-label rifaximin, 73 received double-blind rifaximin $(n=37)$ or placebo $(n=36)$. A total of 1429 bacterial and yeast isolates were identified, of which Bacteroidaceae (36.7\%) and Enterobacteriaceae (33.9\%) were the most common. In the double-blind phase, Clostridium difficile was highly susceptible to rifaximin [minimum inhibitory concentration (MIC) range $0.008-1 \mu \mathrm{g} / \mathrm{mL}$ ] and rifampin (MIC range 0.004-0.25 $\mu \mathrm{g}$ / $\mathrm{mL}$ ). Following double-blind rifaximin treatment, Staphylococcus isolates remained susceptible to rifaximin at all visits $\left(\mathrm{MIC}_{50}\right.$ range $\leq 0.06-32 \mu \mathrm{g} / \mathrm{mL}$ ). Rifaximin exposure was not associated with long-term cross-resistance of Bacteroidaceae, Enterobacteriaceae, and Enterococcaceae to rifampin or nonrifamycin antibiotics tested.

Conclusions In this study, short-term repeat treatment with rifaximin has no apparent long-term effect on stool microbial susceptibility to rifaximin, rifampin, and nonrifamycin antibiotics.

ClinicalTrials.gov Identifier NCT01543178.

Keywords Irritable bowel syndrome $\cdot$ Diarrhea

Microbiology $\cdot$ Rifaximin

\section{Introduction}

Irritable bowel syndrome (IBS) is a chronic, recurrent functional bowel disorder characterized by abdominal pain with alterations in bowel habit $[1,2]$. Subtypes of IBS are 
characterized by stool pattern and include diarrhea-predominant IBS (IBS-D), constipation-predominant IBS, and mixed IBS [1]. The etiology of IBS remains to be fully elucidated; however, IBS is considered a multifactorial condition that is affected by both host (e.g., alterations in gut motility and/or gut microbiota) and environmental factors (e.g., diet) [2]. Indeed, the gut microbiota of patients with IBS-D differ both quantitatively and qualitatively from those of healthy individuals [3-6]. Antibiotics (e.g., rifaximin, neomycin) have been shown to improve symptoms of IBS [7-9]. However, administration of systemic antibiotics may be limited in IBS because of the potential for the development of bacterial antibiotic resistance and adverse events and because of the increased risk of Clostridium difficile infection [10-12].

Rifaximin is an oral nonsystemic gastrointestinal-targeted antibiotic with broad-spectrum activity in vitro against Gram-negative and Gram-positive aerobic and anaerobic bacteria, including $C$. difficile [13-15]. Rifaximin binds the $\beta$ subunit of DNA-dependent RNA polymerase of target bacteria, inhibiting the synthesis of bacterial RNA [13, 16]. Rifaximin has demonstrated efficacy and safety in the treatment of IBS-D and was approved in 2015 by the US Food and Drug Administration for the treatment of IBS-D in adults [17-21]. In two phase 3 identically designed, randomized, double-blind studies [Trial 1 and 2 (TARGET 1 and 2)], a significantly greater percentage of patients with IBS-D receiving rifaximin $550 \mathrm{mg}$ three times daily for 14 days reported adequate relief of global IBS symptoms for $\geq 2$ of the first 4 weeks post-treatment compared with patients receiving placebo (pooled data: 40.7 vs. $31.7 \%$, respectively; $P<0.001$ ) [17]. In a double-blind study evaluating the efficacy and safety of repeat treatment with rifaximin [Trial 3 (TARGET 3)], patients who experienced symptom relapse within 18 weeks of observation after initial response $(\geq 30 \%$ decrease from baseline in mean weekly pain score and $\geq 50 \%$ decrease from baseline in number of days/week with Bristol Stool Scale type 6 or 7 stool for $\geq 2$ of first 4 weeks post-treatment) to open-label rifaximin $550 \mathrm{mg}$ three times a day for 2 weeks were randomized to receive repeat treatments of rifaximin $550 \mathrm{mg}$ three times a day or placebo for 14 days. The percentage of responders to repeat treatment was significantly greater with rifaximin than with placebo (38.1 vs. $31.5 \%, P=0.03$ ) [21].

The safety profile of rifaximin was generally comparable to that of placebo according to the results of a pooled safety analysis of patients with nonconstipation forms of IBS participating in a phase 2 study and Trial 1 and 2 $(n=1932)$ [22]. No patients in the pooled population developed $C$. difficile colitis during dosing. The risk of bacteria developing resistance with rifaximin is thought to be low [10], possibly because of the minimal systemic absorption of rifaximin [23, 24], the requirement of a stable mutation in host cell DNA (in contrast with plasmidbased mechanisms) [25], and the inability of resistant bacteria to effectively colonize the gastrointestinal tract $[26,27]$. Mutations associated with rifaximin resistance following exposure to high concentrations of rifaximin have been shown to occur in approximately $1.0 \times 10^{-8}$ to $2.6 \times 10^{-6}$ bacteria, with some variation among bacterial species $[14,28]$. The aim of this substudy was to examine the susceptibility of stool bacteria to select antibiotics, including rifaximin and rifampin, isolated from a subgroup of patients with IBS-D who participated in Trial 3.

\section{Methods}

\section{Study Design and Patients}

The patient population and the study design of Trial 3 (TARGET 3) have been described previously [21]. Briefly, Trial 3 was a randomized, double-blind, placebocontrolled phase 3 study (ClinicalTrials.gov identifier NCT01543178). Patients aged $\geq 18$ years were eligible to participate if they had a diagnosis of IBS (based on Rome III criteria) and did not experience adequate relief of global IBS symptoms and bloating during a placebo screening phase. Patients were excluded if (after beginning diary assessments) they were taking probiotics or taking rifaximin or any other antibiotic 14 days prior to providing written informed consent [21].

Patients received open-label rifaximin $550 \mathrm{mg}$ three times daily for 2 weeks, followed by a 4 -week treatmentfree follow-up to assess response (improvement in abdominal pain due to IBS $(\geq 30 \%$ reduction from baseline in mean weekly pain score) and stool consistency ( $\geq 50 \%$ reduction from baseline in number of days/week with Bristol Stool Scale type 6 or 7 stool) during $\geq 2$ of the first 4 weeks post-treatment) [21]. Responders continued in the study (nonresponders were withdrawn from further participation) and were monitored during a treatment-free observation phase of up to 18 weeks to determine IBS symptom recurrence (defined as loss of treatment response for either weekly abdominal pain or stool consistency for $\geq 3$ weeks of a consecutive, rolling 4-week period during the 18-week observation phase). Patients with recurrence were randomly assigned (1:1), in a double-blind manner, to two repeat 2-week courses of treatment with rifaximin $550 \mathrm{mg}$ or placebo three times daily, with the two repeat phases separated by 10 weeks. All patients provided written informed consent [21]. 


\section{Collection of Stool Samples}

All patients participating in Trial 3 also consented to provide stool samples. For inclusion in the current substudy, patients were selected using random number generator statistical software. Patients were selected in a manner to incorporate both responders and nonresponders. Prospective fecal culture and susceptibility testing were conducted with stool samples collected from patients prior to (open-label baseline) and after open-label treatment with rifaximin (open-label week 2), and prior to (double-blind baseline) and after the first double-blind course (double-blind week 2), and at the end of the study. Fresh stool samples were collected from patients at study visits when possible; patients who could not provide a fresh stool sample received a stool collection kit for home use with instruction to refrigerate the sample immediately and to deliver it to the clinic as soon as possible. At the clinic, stool samples were divided into 2-mL aliquots in polypropylene cryovials and stored at $\leq-20{ }^{\circ} \mathrm{C}$; samples were then shipped on dry ice for long-term storage at $\leq-70{ }^{\circ} \mathrm{C}$.

Bacteria were isolated from stool by inoculation on tryptic soy agar plates with 5\% sheep's blood to identify aerobic bacteria; the anaerobes $C$. difficile and Bacteroides species were isolated by inoculation of stool samples onto cycloserine cefoxitin fructose agar or Bacteroides bile esculin agar, respectively. Identification of bacteria was achieved by morphology, Gram staining, other tests (catalase, hemolysis), and molecular identification as necessary. The presence of yeast was confirmed by wet mount, and yeast species were identified by the Vitek ${ }^{\circledR} 2$ System (bioMérieux, Inc., Durham, NC, USA); susceptibility testing was not performed on yeast isolates.

\section{Susceptibility Testing}

Bacteria tested for antibiotic susceptibility were from the genera Bacteroides, Citrobacter, Clostridium, Enterobacter, Enterococcus, Escherichia, Klebsiella, Pseudomonas, Serratia, and Staphylococcus. Susceptibility testing was performed with 11 antibiotics using either the broth microdilution method (aerobic bacteria) or the agar dilution method (anaerobic bacteria). Aerobic bacteria were tested with rifaximin, rifampin, ceftazidime, ceftriaxone, ciprofloxacin, imipenem, meropenem, and piperacillin/tazobactam. Anaerobic bacteria were tested with rifaximin, rifampin, fidaxomicin, metronidazole, and vancomycin.

\section{Data Analysis}

Each bacterial species isolated and tested was grouped into a family [i.e., Bacteroidaceae, Clostridiaceae, Enterobacteriaceae (including Escherichia, Klebsiella, Proteus, Enterobacter, and Citrobacter species), Enterococcaceae, Pseudomonadaceae, or Staphylococcaceae]. The minimum inhibitory concentration (MIC) at which $\geq 50 \%$ of isolates were inhibited was the $\mathrm{MIC}_{50}$ for the population, and the MIC at which $\geq 90 \%$ of isolates were inhibited was the $\mathrm{MIC}_{90}$ for the population. Clinical and Laboratory Standards Institute (CLSI) break points for each species were used to interpret MIC data [29]. For antibiotics with defined susceptible and intermediate categories, the susceptible classification was used for these analyses. For antibiotics without established CLSI break points, ranges for in vitro sensitivity were used if provided on the package insert. In all other cases (i.e., antibiotics without CLSI defined break points, in vitro susceptibility not provided on package insert), MIC values at or above the highest dilution tested in the MIC panel were considered resistant, and MIC values below the highest dilution were considered susceptible. The follow-up period was variable; therefore, for purposes of data analysis, the follow-up visits were combined into 4-week periods.

\section{Results}

\section{Patient Population}

A total of 103 patients were randomly selected for inclusion in the stool microbiota substudy; this was a subgroup of patients included in the Trial 3 study [21]. Patients were mostly white $(82.5 \%)$, mostly female (73.8\%), had a mean age of 47.9 years (range 19-85 years), and had a baseline average of 3.5 daily bowel movements. Of the 103 patients who received openlabel rifaximin, 73 also participated in the double-blind phase (rifaximin, $n=37$; placebo, $n=36$ ). In the double-blind phase, a similar percentage of patients in the rifaximin and placebo groups, respectively, were female (70.3 and 72.2\%) and had a similar frequency of daily bowel movements (3.1 and 2.9). Patients in the rifaximin and placebo groups had similar mean ages, respectively, of 46.9 years (range 22-85 years), and 47.7 years (range 19-74 years); however, the percentage of white patients was lower in the rifaximin group $(75.7 \%)$ than in the placebo group $(88.9 \%)$.

A total of 1429 bacterial and yeast isolates were identified from stool samples; the most common isolates were members of the families Bacteroidaceae (525 [36.7\%]) and Enterobacteriaceae (484 [33.9\%]; Table 1). A small number of Clostridiaceae (22 [1.5\%]) and Pseudomonadaceae (4 [0.3\%]) isolates were cultured. A total of 17 yeast isolates from 14 patients were identified (1.2\%).

\section{Rifaximin and Rifampin Susceptibility}

\section{Open-Label Phase}

In the open-label phase, 366 Bacteroides isolates were identified (Table S1). The range of MIC values for 
Table 1 Most abundant isolates from stool cultures $(N=1429)$

\begin{tabular}{lcc}
\hline Bacteria family & Counts & Percentage of total \\
\hline Bacteroidaceae & 525 & 36.7 \\
Bacteroides vulgatus & 178 & 12.5 \\
Bacteroides ovatus & 114 & 8.0 \\
Bacteroides fragilis & 102 & 7.1 \\
Enterobacteriaceae & 484 & 33.9 \\
Escherichia coli & 337 & 23.6 \\
Klebsiella pneumoniae & 72 & 5.0 \\
Enterococcaceae & 286 & 20.0 \\
Enterococcus faecalis & 117 & 8.2 \\
Enterococcus faecium & 90 & 6.3 \\
Staphylococcaceae & 91 & 6.4 \\
Staphylococcus aureus & 25 & 1.7 \\
\hline
\end{tabular}

Species presented in table do not represent all isolates that were cultured from stool. The isolates included in the table represent bacterial families that are $\geq 5 \%$ of the total number of isolates and bacteria of clinical importance

rifaximin and rifampin was wide at each visit and across visits. The $\mathrm{MIC}_{50}$ and $\mathrm{MIC}_{90}$ values for rifaximin increased from baseline, although susceptible isolates were still observed at week 2 and weeks $7-32$. The $\mathrm{MIC}_{50}$ values for rifampin increased from baseline to week 2 and remained high through week 23 , when the $\mathrm{MIC}_{50}$ value returned to the baseline level.

A total of $14 \mathrm{C}$. difficile isolates were identified; all isolates were highly susceptible to rifaximin (MIC range $0.008-0.12 \mu \mathrm{g} / \mathrm{mL}$ ) across visits. Similarly, most $C$. diffcile isolates $(n=14)$ were highly susceptible to rifampin (MIC range $0.002-0.015 \mu \mathrm{g} / \mathrm{mL}$ ) across all visits, with one exception. The $C$. difficile isolate from one patient had a MIC of $16 \mu \mathrm{g} / \mathrm{mL}$ at week $\geq 23$; however, this isolate was sensitive to all other antibiotics tested, including rifaximin.

A total of 328 Enterobacteriaceae isolates were identified (Table S2). MIC 50 and $\mathrm{MIC}_{90}$ values for rifaximin increased from baseline to week 2 and remained higher until weeks 19-22, when $\mathrm{MIC}_{50}$ and $\mathrm{MIC}_{90}$ values decreased for the rest of the study. The baseline rifampin MIC range increased to a maximum MIC at week 2.

In addition, susceptibility of Enterococcaceae to rifaximin was consistent throughout the open-label phase (Table S3). Only three Pseudomonas isolates were identified in the open-label phase of the study, all in the followup period. Staphylococcaceae isolates were highly susceptible to rifaximin and rifampin at baseline; at week 2, the rifaximin and rifampin $\mathrm{MIC}_{50}$ and $\mathrm{MIC}_{90}$ values increased (Table S4). Rifaximin $\mathrm{MIC}_{50}$ levels recovered to baseline levels at week $7 ; \mathrm{MIC}_{90}$ levels recovered to baseline levels at week 23 .

\section{Double-Blind Phase}

A total of 107 and 129 Bacteroides isolates were identified for rifaximin susceptibility testing in samples from patients receiving double-blind rifaximin or placebo, respectively; 121 and 129 Bacteroides isolates were identified for rifampin susceptibility testing in samples from patients receiving double-blind rifaximin or placebo, respectively. Bacteroides isolates demonstrated a wide range of susceptibility to rifaximin and rifampin for both the doubleblind rifaximin and placebo groups, respectively (Table 2). There were no apparent differences in susceptibility of Bacteroides to rifaximin or rifampin in the double-blind rifaximin or placebo groups. Rifaximin $\mathrm{MIC}_{50}$ values decreased compared with baseline at week $\geq 23$ following double-blind rifaximin or placebo treatment.

No differences in susceptibility to rifaximin and rifampin were observed for $C$. difficile isolates identified in the double-blind rifaximin (five isolates tested) and placebo groups (nine isolates tested). $C$. difficile was highly susceptible to rifaximin (MIC range $0.008-1 \mu \mathrm{g} / \mathrm{mL}$ ) and rifampin (MIC range $0.004-0.25 \mu \mathrm{g} / \mathrm{mL}$ ). No C. difficile isolates were isolated beyond week 2 for the double-blind rifaximin group for rifaximin susceptibility testing.

Susceptibility of Enterobacteriaceae isolates to rifaximin and rifampin was consistent between the double-blind rifaximin and placebo groups (Table 3). In the doubleblind rifaximin group, rifaximin $\mathrm{MIC}_{50}$ values increased from baseline to week 2, then decreased to baseline levels from weeks 7 to 22 of the follow-up period. In the doubleblind placebo group, rifaximin $\mathrm{MIC}_{50}$ values were unchanged from baseline through the follow-up period. Rifampin $\mathrm{MIC}_{50}$ values ranged between 8 and $16 \mu \mathrm{g} / \mathrm{mL}$ for the double-blind rifaximin group and were $16 \mu \mathrm{g} / \mathrm{mL}$ for all visits in the placebo group.

The susceptibility of Enterococcaceae to rifaximin and rifampin was consistent between the double-blind rifaximin and placebo groups (Table 4). Differences were observed in the percentages of rifampin-resistant Enterococcus isolates cultured from the stools of patients receiving double-blind placebo and rifaximin at baseline (53 vs. 39\%, respectively), week 2 (37 vs. 50\%), and during the follow-up period (64 vs. 52\%).

A total of three Pseudomonadaceae isolates were identified and evaluated for susceptibility to rifaximin and rifampin. The one isolate from the double-blind rifaximin group had rifaximin and rifampin MICs of 16 and $32 \mu \mathrm{g} /$ $\mathrm{mL}$, respectively. The two isolates from the placebo group had a baseline rifaximin and rifampin MIC of $16 \mu \mathrm{g} / \mathrm{mL}$ and had rifaximin and rifampin MICs of 4 and $8 \mu \mathrm{g} / \mathrm{mL}$, respectively, during the follow-up period (weeks 19-22). 
Table 2 Susceptibility of Bacteroidaceae to rifaximin or rifampin in the double-blind phase of the study

\begin{tabular}{|c|c|c|c|c|c|c|c|c|}
\hline \multirow[t]{2}{*}{ Timepoint $^{\mathrm{a}}$ (patients) } & \multicolumn{4}{|c|}{ Rifaximin } & \multicolumn{4}{|c|}{ Rifampin } \\
\hline & $\begin{array}{l}\text { Isolates } \\
(n)\end{array}$ & $\begin{array}{l}\text { MIC range } \\
(\mu \mathrm{g} / \mathrm{mL})\end{array}$ & $\begin{array}{l}\mathrm{MIC}_{50} \\
(\mu \mathrm{g} / \mathrm{mL})\end{array}$ & $\begin{array}{l}\mathrm{MIC}_{90} \\
(\mu \mathrm{g} / \mathrm{mL})\end{array}$ & $\begin{array}{l}\text { Isolates } \\
(n)\end{array}$ & $\begin{array}{l}\text { MIC range } \\
(\mu \mathrm{g} / \mathrm{mL})\end{array}$ & $\begin{array}{l}\mathrm{MIC}_{50} \\
(\mu \mathrm{g} / \mathrm{mL})\end{array}$ & $\begin{array}{l}\mathrm{MIC}_{90} \\
(\mu \mathrm{g} / \mathrm{mL})\end{array}$ \\
\hline \multicolumn{9}{|l|}{ Double-blind rifaximin } \\
\hline Baseline $(n=37)$ & 47 & $0.12->1024$ & $>1024$ & $>1024$ & 47 & $0.12->64$ & $>64$ & $>64$ \\
\hline Week $2^{\mathrm{b}}(n=36)$ & 27 & $0.12->1024$ & $>1024$ & $>1024$ & 42 & $0.12->64$ & $>64$ & $>64$ \\
\hline Weeks $7-10(n=1)$ & 2 & $512->1024$ & NA & NA & 2 & $32->64$ & NA & NA \\
\hline Weeks 19-22 $(n=3)$ & 4 & $0.5->1024$ & $>1024$ & $>1024$ & 4 & $0.12->64$ & $>64$ & $>64$ \\
\hline Week $\geq 23(n=30)$ & 27 & $0.12->1024$ & 256 & $>1024$ & 26 & $0.03->64$ & $>64$ & $>64$ \\
\hline \multicolumn{9}{|l|}{ Double-blind placebo } \\
\hline Baseline $(n=36)$ & 45 & $0.25->1024$ & 512 & $>1024$ & 45 & $0.06->64$ & $>64$ & $>64$ \\
\hline Week $2^{\mathrm{b}}(n=36)$ & 42 & $0.25->1024$ & 512 & $>1024$ & 42 & $0.12->64$ & $>64$ & $>64$ \\
\hline Weeks $11-14(n=1)$ & 2 & $0.5-256$ & NA & NA & 2 & $0.25->64$ & NA & NA \\
\hline Weeks $15-18(n=3)$ & 3 & $128->1024$ & $>1024$ & $>1024$ & 3 & $32->64$ & $>64$ & $>64$ \\
\hline Weeks 19-22 $(n=6)$ & 9 & $0.25->1024$ & 0.5 & $>1024$ & 9 & $0.12->64$ & 0.12 & $>64$ \\
\hline Week $\geq 23(n=26)$ & 28 & $0.12->1024$ & 128 & $>1024$ & 28 & $0.03->64$ & 16 & $>64$ \\
\hline
\end{tabular}

$M I C$ minimum inhibitory concentration, $M I C_{50}$ MIC at which $\geq 50 \%$ of isolates were inhibited, $M I C_{90}$ MIC at which $\geq 90 \%$ of isolates were inhibited, $N A$ not applicable

${ }^{\text {a }}$ Follow-up period was variable; therefore, the follow-up visits were grouped into 4-week periods to determine whether there was an effect of time on antibiotic susceptibility of isolates

b End of 2-week rifaximin or placebo treatment

Table 3 Susceptibility of Enterobacteriaceae to rifaximin or rifampin in the double-blind phase of the study

\begin{tabular}{|c|c|c|c|c|c|c|c|c|}
\hline \multirow[t]{2}{*}{ Timepoint $^{\mathrm{a}}$ (patients) } & \multicolumn{4}{|c|}{ Rifaximin } & \multicolumn{4}{|c|}{ Rifampin } \\
\hline & $\begin{array}{l}\text { Isolates } \\
(n)\end{array}$ & $\begin{array}{l}\text { MIC range } \\
(\mu \mathrm{g} / \mathrm{mL})\end{array}$ & $\begin{array}{l}\mathrm{MIC}_{50} \\
(\mu \mathrm{g} / \mathrm{mL})\end{array}$ & $\begin{array}{l}\mathrm{MIC}_{90} \\
(\mu \mathrm{g} / \mathrm{mL})\end{array}$ & $\begin{array}{l}\text { Isolates } \\
(n)\end{array}$ & $\begin{array}{l}\text { MIC range } \\
(\mu \mathrm{g} / \mathrm{mL})\end{array}$ & $\begin{array}{l}\mathrm{MIC}_{50} \\
(\mu \mathrm{g} / \mathrm{mL})\end{array}$ & $\begin{array}{l}\mathrm{MIC}_{90} \\
(\mu \mathrm{g} / \mathrm{mL})\end{array}$ \\
\hline \multicolumn{9}{|l|}{ Double-blind rifaximin } \\
\hline Baseline $(n=37)$ & 37 & $8->128$ & 16 & $>128$ & 37 & $4-64$ & 16 & 32 \\
\hline Week $2^{\mathrm{b}}(n=36)$ & 30 & $8->128$ & 64 & $>128$ & 30 & $4->128$ & 16 & $>128$ \\
\hline Weeks 7-10 $(n=1)$ & 4 & $8-64$ & 16 & 64 & 4 & $8-32$ & 8 & 32 \\
\hline Weeks $19-22(n=3)$ & 3 & $8-16$ & 16 & 16 & 3 & $4-16$ & 8 & 16 \\
\hline Week $\geq 23(n=30)$ & 34 & $8->128$ & 32 & 64 & 34 & $8->128$ & 16 & 32 \\
\hline \multicolumn{9}{|l|}{ Double-blind placebo } \\
\hline Baseline $(n=36)$ & 38 & $4->128$ & 32 & $>128$ & 38 & $4->128$ & 16 & $>128$ \\
\hline Week $2^{\mathrm{b}}(n=36)$ & 41 & $4->128$ & 32 & $>128$ & 41 & $4->128$ & 16 & 128 \\
\hline Weeks 11-14 $(n=1)$ & 1 & 8 & NA & NA & 1 & 8 & NA & NA \\
\hline Weeks $15-18(n=3)$ & 2 & $16-32$ & NA & NA & 2 & $8-16$ & NA & NA \\
\hline Weeks 19-22 $(n=6)$ & 9 & $4->128$ & 32 & $>128$ & 9 & $1-128$ & 16 & 128 \\
\hline Week $\geq 23(n=26)$ & 32 & $4->128$ & 32 & $>128$ & 32 & $8->128$ & 16 & 32 \\
\hline
\end{tabular}

$M I C$ minimum inhibitory concentration, $M I C_{50}$ MIC at which $\geq 50 \%$ of isolates were inhibited, $M I C_{90}$ MIC at which $\geq 90 \%$ of isolates were inhibited, $N A$ not applicable

${ }^{a}$ Follow-up period was variable; therefore, the follow-up visits were grouped into 4-week periods to determine whether there was an effect of time on antibiotic susceptibility of isolates

${ }^{\mathrm{b}}$ End of 2-week rifaximin or placebo treatment

Staphylococcal isolates remained susceptible to rifaximin and rifampin at all visits in the double-blind placebo group. In the double-blind rifaximin group, the rifaximin
$\mathrm{MIC}_{50}$ increased from baseline to week $2(\leq 0.06-32 \mu \mathrm{g} /$ $\mathrm{mL}$, respectively), but the five isolates evaluated in the follow-up period had rifaximin MICs $\leq 0.06 \mu \mathrm{g} / \mathrm{mL}$ 
Table 4 Susceptibility of Enterococcaceae to rifaximin or rifampin in the double-blind phase of the study

\begin{tabular}{|c|c|c|c|c|c|c|c|c|}
\hline \multirow[t]{2}{*}{ Timepoint $^{\mathrm{a}}$ (patients) } & \multicolumn{4}{|c|}{ Rifaximin } & \multicolumn{4}{|c|}{ Rifampin } \\
\hline & $\begin{array}{l}\text { Isolates } \\
(n)\end{array}$ & $\begin{array}{l}\text { MIC range } \\
(\mu \mathrm{g} / \mathrm{mL})\end{array}$ & $\begin{array}{l}\mathrm{MIC}_{50} \\
(\mu \mathrm{g} / \mathrm{mL})\end{array}$ & $\begin{array}{l}\mathrm{MIC}_{90} \\
(\mu \mathrm{g} / \mathrm{mL})\end{array}$ & $\begin{array}{l}\text { Isolates } \\
(n)\end{array}$ & $\begin{array}{l}\text { MIC range } \\
(\mu \mathrm{g} / \mathrm{mL})\end{array}$ & $\begin{array}{l}\mathrm{MIC}_{50} \\
(\mu \mathrm{g} / \mathrm{mL})\end{array}$ & $\begin{array}{l}\mathrm{MIC}_{90} \\
(\mu \mathrm{g} / \mathrm{mL})\end{array}$ \\
\hline \multicolumn{9}{|l|}{ Double-blind rifaximin } \\
\hline Baseline $(n=37)$ & 33 & $\leq 0.06-16$ & 2 & 16 & 33 & $\leq 0.06-16$ & 1 & 8 \\
\hline Week $2^{\mathrm{b}}(n=36)$ & 20 & $\leq 0.06-32$ & 4 & 16 & 20 & $\leq 0.06-32$ & 2 & 16 \\
\hline Weeks $7-10(n=1)$ & 1 & 32 & NA & NA & 1 & 8 & NA & NA \\
\hline Weeks 19-22 $(n=3)$ & 0 & NA & NA & NA & 0 & NA & NA & NA \\
\hline Week $\geq 23(n=30)$ & 21 & $\leq 0.06-32$ & 4 & 32 & 21 & $\leq 0.06-32$ & 4 & 16 \\
\hline \multicolumn{9}{|l|}{ Double-blind placebo } \\
\hline Baseline $(n=36)$ & 19 & $\leq 0.06-32$ & 4 & 32 & 19 & $\leq 0.06-16$ & 4 & 16 \\
\hline Week $2^{\mathrm{b}}(n=36)$ & 30 & $\leq 0.06-32$ & 2 & 16 & 30 & $\leq 0.06-32$ & 2 & 16 \\
\hline Weeks $11-14(n=1)$ & 0 & NA & NA & NA & 0 & NA & NA & NA \\
\hline Weeks $15-18(n=3)$ & 1 & 2 & NA & NA & 1 & 1 & NA & NA \\
\hline Weeks 19-22 $(n=6)$ & 7 & $0.5-16$ & 4 & 16 & 7 & $0.15-16$ & 4 & 16 \\
\hline Week $\geq 23(n=26)$ & 17 & $\leq 0.06-32$ & 16 & 32 & 17 & $\leq 0.06-32$ & 8 & 32 \\
\hline
\end{tabular}

$M I C$ minimum inhibitory concentration, $M I C_{50}$ MIC at which $\geq 50 \%$ of isolates were inhibited, $M I C_{90}$ MIC at which $\geq 90 \%$ of isolates were inhibited, $N A$ not applicable

${ }^{\text {a }}$ Follow-up period was variable; therefore, the follow-up visits were grouped into 4-week periods to determine whether there was an effect of time on antibiotic susceptibility of isolates

${ }^{\mathrm{b}}$ End of 2-week rifaximin treatment

Table 5 Susceptibility of Staphylococcaceae to rifaximin or rifampin in the double-blind phase of the study

\begin{tabular}{|c|c|c|c|c|c|c|c|c|}
\hline \multirow[t]{2}{*}{ Timepoint $^{\mathrm{a}}$ (patients) } & \multicolumn{4}{|c|}{ Rifaximin } & \multicolumn{4}{|c|}{ Rifampin } \\
\hline & $\begin{array}{l}\text { Isolates } \\
(n)\end{array}$ & $\begin{array}{l}\text { MIC range } \\
(\mu \mathrm{g} / \mathrm{mL})\end{array}$ & $\begin{array}{l}\mathrm{MIC}_{50} \\
(\mu \mathrm{g} / \mathrm{mL})\end{array}$ & $\begin{array}{l}\text { MIC }_{90} \\
(\mu \mathrm{g} / \mathrm{mL})\end{array}$ & $\begin{array}{l}\text { Isolates } \\
(n)\end{array}$ & $\begin{array}{l}\text { MIC range } \\
(\mu \mathrm{g} / \mathrm{mL})\end{array}$ & $\begin{array}{l}\mathrm{MIC}_{50} \\
(\mu \mathrm{g} / \mathrm{mL})\end{array}$ & $\begin{array}{l}\mathrm{MIC}_{90} \\
(\mu \mathrm{g} / \mathrm{mL})\end{array}$ \\
\hline \multicolumn{9}{|l|}{ Double-blind rifaximin } \\
\hline Baseline $(n=37)$ & 9 & $\leq 0.06-64$ & $\leq 0.06$ & 64 & 9 & $\leq 0.06->128$ & $\leq 0.06$ & $>128$ \\
\hline Week $2^{\mathrm{b}}(n=36)$ & 10 & $\leq 0.06-64$ & 32 & 64 & 10 & $\leq 0.06->128$ & $>128$ & $>128$ \\
\hline Weeks 7-10 $(n=1)$ & 0 & NA & NA & NA & 0 & NA & NA & NA \\
\hline Weeks 19-22 $(n=3)$ & 2 & $\leq 0.06$ & NA & NA & 2 & $\leq 0.06$ & NA & NA \\
\hline Week $\geq 23(n=30)$ & 3 & $\leq 0.06$ & $\leq 0.06$ & $\leq 0.06$ & 3 & $\leq 0.06$ & $\leq 0.06$ & $\leq 0.06$ \\
\hline \multicolumn{9}{|l|}{ Double-blind placebo } \\
\hline Baseline $(n=36)$ & 8 & $\leq 0.06-32$ & $\leq 0.06$ & 32 & 8 & $\leq 0.06->128$ & $\leq 0.06$ & $>128$ \\
\hline Week $2^{\mathrm{b}}(n=36)$ & 7 & $\leq 0.06-64$ & $\leq 0.06$ & 64 & 7 & $\leq 0.06->128$ & $\leq 0.06$ & $>128$ \\
\hline Weeks $11-14(n=1)$ & 0 & NA & NA & NA & 0 & NA & NA & NA \\
\hline Weeks $15-18(n=3)$ & 0 & NA & NA & NA & 0 & NA & NA & NA \\
\hline Weeks 19-22 $(n=6)$ & 1 & $\leq 0.06$ & NA & NA & 1 & $\leq 0.06$ & NA & NA \\
\hline Week $\geq 23(n=26)$ & 6 & $\leq 0.06$ & $\leq 0.06$ & $\leq 0.06$ & 6 & $\leq 0.06$ & $\leq 0.06$ & $\leq 0.06$ \\
\hline
\end{tabular}

MIC minimum inhibitory concentration, $M I C_{50}$ MIC at which $\geq 50 \%$ of isolates were inhibited, $M I C_{90}$ MIC at which $\geq 90 \%$ of isolates were inhibited, $N A$ not applicable

${ }^{a}$ Follow-up period was variable; therefore, the follow-up visits were grouped into 4-week periods to determine whether there was an effect of time on antibiotic susceptibility of isolates

${ }^{b}$ End of 2-week rifaximin treatment

(Table 5). At baseline, 11 and $13 \%$ of staphylococcal isolates in the rifaximin and placebo groups, respectively, were rifampin resistant (MIC $\geq 4 \mu \mathrm{g} / \mathrm{mL}$ ); at week $2,70 \%$ of staphylococcal isolates in the rifaximin group were rifampin resistant. However, repeat treatment with rifaximin did not have an apparent effect on the long-term 
susceptibility of staphylococcal isolates to rifampin, as isolates recovered sensitivity to rifampin in the follow-up period $(\leq 0.06 \mu \mathrm{g} / \mathrm{mL})$.

\section{Other Antibiotic Susceptibility}

In the open-label phase, there was no apparent cross-resistance of Bacteroidaceae (Table S5), Enterobacteriaceae (Table S6), and Enterococcaceae (Table S7) to nonrifamycin antibiotics following exposure to rifaximin. The small number of Clostridiaceae, Pseudomonadaceae, and Staphylococcaceae isolates available limited $\mathrm{MIC}_{50}$ analyses at the time points assessed with antibiotics tested.

In the double-blind phase, there was no apparent crossresistance of Bacteroidaceae (Table S8), Enterobacteriaceae (Table S9), and Enterococcaceae (Table S10) to nonrifamycin antibiotics following rifaximin exposure. The small number of Clostridiaceae, Pseudomonadaceae, and Staphylococcaceae isolates available limited $\mathrm{MIC}_{50}$ analyses at the time points assessed with the antibiotics tested.

\section{Discussion}

In the current study, short-term repeat treatment with rifaximin in patients with IBS-D was not associated with clinically meaningful resistance to rifaximin, rifampin, and nonrifamycin antibiotics tested in the long term. These findings support previous reports about rifaximin treatment of patients with hepatic encephalopathy or travelers' diarrhea, suggesting that the potential for development of cross-resistance to nonrifamycin antibiotics following treatment with rifaximin is low [30-34].

Overall, bacterial isolates were susceptible to rifaximin and rifampin during the open-label and double-blind phases of the study. Increases in the $\mathrm{MIC}_{50}$ or the $\mathrm{MIC}_{90}$ of rifaximin against Bacteroides were within baseline MIC ranges during both the open-label and the double-blind phases of the study and within the MIC ranges previously reported in the literature [35]. The $\mathrm{MIC}_{50}$ of rifaximin with Enterobacteriaceae increased at week 2 in both the openlabel and the double-blind phases of this study but recovered to baseline levels in the follow-up period; this observation is consistent with the rapid elimination of rifaximin-resistant bacteria, particularly aerobes, from the stool [26]. Transient increases in rifaximin and rifampin $\mathrm{MIC}_{50}$ values for staphylococcal isolates were observed after treatment with open-label rifaximin (week 2) and after repeat treatment with double-blind rifaximin (week 2); however, MIC values for rifaximin and rifampin returned to baseline levels as the duration of time post-rifaximin exposure increased (week $\geq 23$ ). The transient increases observed in the short term do not appear to be clinically harmful. Yeast growth can occur following treatment with some antibiotics [36, 37]; however, the small number of yeast isolates identified supports a previous finding that rifaximin does not create an environment favorable to yeast growth [27].

A previous study has shown that some patients with IBS may be predisposed to infection with $C$. difficile, although isolates from these patients were susceptible to the antibiotics tested [38]. One limitation of the current study was the lack of consistency in antimicrobial susceptibility testing for bacterial families with a small number of isolates identified (i.e., Clostridiaceae, Pseudomonadaceae). The human gut microbiome is thought to contain at least 1200 different microorganisms; thus, an additional limitation of this study was the limited number of bacterial species examined for resistance relative to the quantity and diversity of microbes in the human gut microbiome [39]. Finally, the use of fecal samples, while noninvasive, may not be entirely representative of the composition and, possibly, activity of the gut microbiota in vivo [40]. Indeed, the bacterial composition of fecal and colonic mucosa differed significantly in both healthy individuals $(P=0.0001)$ and patients with IBS-D $(P=0.001)$.

The large number of patients with prospectively collected stool samples available for microbial susceptibility testing strengthens study results. Potential limitations of the study include the small number of isolates collected from some bacterial families, thus limiting potential robustness of the susceptibility testing, uncertainty as to whether this patient sampling was representative of a general population with IBS-D, and the lack of evaluation of the potential relationship between susceptibility profiles and clinical response observed in Trial 3.

In conclusion, because of the potential for chronicity of IBS-D symptoms, patients may require repeat treatment with rifaximin for the management of symptoms [2]. Based on the results of this prospective study, there is no evidence of long-term clinically relevant antibiotic resistance with repeated courses of rifaximin for the treatment of IBS-D.

Acknowledgments Technical editorial assistance was provided, under the direction of the authors, by Mary Beth Moncrief, Ph.D., and Sophie Bolick, Ph.D., Synchrony Medical Communications, LLC, West Chester, PA, USA. Funding for this support was provided by Salix Pharmaceuticals, Bridgewater, NJ, USA.

Author's contributions Dr. Pimentel contributed to the study design, participated in the conduct of the study, analyzed data, and critically reviewed and edited the manuscript. Dr. Cash contributed to the study design, analyzed data, and critically reviewed and edited the manuscript. Dr. Lembo contributed to the study design, participated in the conduct of the study, analyzed data, and critically reviewed and edited the manuscript. Dr. Schoenfeld contributed to the study design, participated in the conduct of the study, analyzed data, and critically reviewed and edited the manuscript. Drs. Wolf and Israel analyzed data and critically reviewed and edited the manuscript. All of the 
authors approved the final version of the manuscript, including the authorship list.

\section{Compliance with ethical standards}

Conflict of interest M. Pimentel reports serving as a consultant for and receiving research funding from Salix Pharmaceuticals. In addition, Cedars-Sinai Medical Center has a licensing agreement with Salix Pharmaceuticals. B.D. Cash reports serving as a speaker, consultant, and an advisory board member for Salix Pharmaceuticals. A. Lembo reports serving as a consultant and an advisory board member for Salix Pharmaceuticals, Ironwood Pharmaceuticals, Inc., Allergan plc, Ardelyx, and Valeant Pharmaceuticals. R.A. Wolf is a former employee of Salix Pharmaceuticals. R.J. Israel is an employee of Salix Pharmaceuticals or its affiliates. P. Schoenfeld reports serving as a consultant and advisory board member for Salix Pharmaceuticals, Ironwood Pharmaceuticals, Allergan, and Commonwealth Laboratories, a division of Valeant Pharmaceuticals North America LLC.

Ethical standards All procedures performed in the study were in accordance with the International Standard of Good Clinical Practice procedures and with the principles of the Declaration of Helsinki (1964) and relevant amendments. The protocol was approved by all institutional review boards and ethics committees at participating sites.

\section{References}

1. Lacy BE, Mearin F, Chang L, et al. Bowel disorders. Gastroenterology. 2016;150:1393-1407.

2. Chey WD, Kurlander J, Eswaran S. Irritable bowel syndrome: a clinical review. JAMA. 2015;313:949-958.

3. Carroll IM, Chang YH, Park J, Sartor RB, Ringel Y. Luminal and mucosal-associated intestinal microbiota in patients with diarrheapredominant irritable bowel syndrome. Gut Pathog. 2010;2:19.

4. Shankar V, Homer D, Rigsbee L, et al. The networks of human gut microbe-metabolite associations are different between health and irritable bowel syndrome. ISME J. 2015;9:1899-1903.

5. Rigsbee L, Agans R, Shankar V, et al. Quantitative profiling of gut microbiota of children with diarrhea-predominant irritable bowel syndrome. Am J Gastroenterol. 2012;107:1740-1751.

6. Rajilic-Stojanovic M, Biagi E, Heilig HG, et al. Global and deep molecular analysis of microbiota signatures in fecal samples from patients with irritable bowel syndrome. Gastroenterology. 2011;141:1792-1801.

7. Ford AC, Moayyedi P, Lacy BE, et al. American College of Gastroenterology monograph on the management of irritable bowel syndrome and chronic idiopathic constipation. Am J Gastroenterol. 2014;109:S2-S26.

8. Pimentel M, Chow EJ, Lin HC. Normalization of lactulose breath testing correlates with symptom improvement in irritable bowel syndrome. a double-blind, randomized, placebo-controlled study. Am J Gastroenterol. 2003;98:412-419.

9. Pimentel M, Chatterjee S, Chow EJ, Park S, Kong Y. Neomycin improves constipation-predominant irritable bowel syndrome in a fashion that is dependent on the presence of methane gas: subanalysis of a double-blind randomized controlled study. Dig Dis Sci. 2006;51:1297-1301.

10. Basseri RJ, Weitsman S, Barlow GM, Pimentel M. Antibiotics for the treatment of irritable bowel syndrome. Gastroenterol Hepatol. 2011;7:455-493.

11. Cash BD. Emerging role of probiotics and antimicrobials in the management of irritable bowel syndrome. Curr Med Res Opin. 2014;30:1405-1415.
12. Blondeau JM. What have we learned about antimicrobial use and the risks for Clostridium difficile-associated diarrhoea? $J$ Antimicrob Chemother. 2009;63:238-242.

13. Jiang ZD, DuPont HL. Rifaximin: in vitro and in vivo antibacterial activity-a review. Chemotherapy. 2005;51:67-72.

14. Marchese A, Salerno A, Pesce A, Debbia EA, Schito GC. In vitro activity of rifaximin, metronidazole and vancomycin against Clostridium difficile and the rate of selection of spontaneously resistant mutants against representative anaerobic and aerobic bacteria, including ammonia-producing species. Chemotherapy. 2000;46:253-266.

15. Gillis JC, Brogden RN. Rifaximin. A review of its antibacterial activity, pharmacokinetic properties and therapeutic potential in conditions mediated by gastrointestinal bacteria. Drugs. 1995;49:467-484.

16. Xifaxan ${ }^{\circledR}$ (rifaximin) tablets, for oral use [package insert]. Bridgewater, NJ: Salix Pharmaceuticals; 2015.

17. Pimentel M, Lembo A, Chey WD, et al. Rifaximin therapy for patients with irritable bowel syndrome without constipation. $N$ Engl J Med. 2011;364:22-32.

18. Meyrat P, Safroneeva E, Schoepfer AM. Rifaximin treatment for the irritable bowel syndrome with a positive lactulose hydrogen breath test improves symptoms for at least 3 months. Aliment Pharmacol Ther. 2012;36:1084-1093.

19. Pimentel M, Park S, Mirocha J, Kane SV, Kong Y. The effect of a nonabsorbed oral antibiotic (rifaximin) on the symptoms of the irritable bowel syndrome: a randomized trial. Ann Intern Med. 2006;145:557-563.

20. Pimentel M, Morales W, Chua K, et al. Effects of rifaximin treatment and retreatment in nonconstipated IBS subjects. Dig Dis Sci. 2011;56:2067-2072.

21. Lembo A, Pimentel M, Rao SS, et al. Repeat treatment with rifaximin is safe and effective in patients with diarrhea-predominant irritable bowel syndrome. Gastroenterology. 2016;151:1113-1121.

22. Schoenfeld P, Pimentel M, Chang L, et al. Safety and tolerability of rifaximin for the treatment of irritable bowel syndrome without constipation: a pooled analysis of randomised, double-blind, placebo-controlled trials. Aliment Pharmacol Ther. 2014;39:1161-1168.

23. Taylor DN, McKenzie R, Durbin A, Carpenter C, Haake R, Bourgeois AL. Systemic pharmacokinetics of rifaximin in volunteers with shigellosis. Antimicrob Agents Chemother. 2008;52:1179-1181.

24. Descombe JJ, Dubourg D, Picard M, Palazzini E. Pharmacokinetic study of rifaximin after oral administration in healthy volunteers. Int J Clin Pharmacol Res. 1994;14:51-56.

25. Kothary V, Scherl EJ, Bosworth B, et al. Rifaximin resistance in Escherichia coli associated with inflammatory bowel disease correlates with prior rifaximin use, mutations in $r p o B$, and activity of phe-arg-b-naphthylamide-inhibitable efflux pumps. Antimicrob Agents Chemother. 2013;57:811-817.

26. De Leo C, Eftimiadi C, Schito GC. Rapid disappearance from the intestinal tract of bacteria resistant to rifaximin. Drugs Exp Clin Res. 1986;12:979-981.

27. Brigidi P, Swennen E, Rizzello F, Bozzolasco M, Matteuzzi D. Effects of rifaximin administration on the intestinal microbiota in patients with ulcerative colitis. J Chemother. 2002;14:290-295.

28. Ruiz J, Mensa L, Pons MJ, Vila J, Gascon J. Development of Escherichia coli rifaximin-resistant mutants: frequency of selection and stability. J Antimicrob Chemother. 2008;61:1016-1019.

29. Clinical and Laboratory Standards Institute. Performance standards for antimicrobial susceptibility testing; twenty-fourth informational supplement, CLSI document M100-S24. Wayne, PA; 2014. 
30. Mullen KD, Sanyal AJ, Bass NM, et al. Rifaximin is safe and well tolerated for long-term maintenance of remission from overt hepatic encephalopathy. Clin Gastroenterol Hepatol. 2014;12:1390-1397.

31. Bass NM, Mullen KD, Sanyal A, et al. Rifaximin treatment in hepatic encephalopathy. N Engl J Med. 2010;362:1071-1081.

32. Bajaj JS, Barrett AC, Bortey E, Paterson C, Forbes WP. Prolonged remission from hepatic encephalopathy with rifaximin: results of a placebo crossover analysis. Aliment Pharmacol Ther. 2015;41:39-45.

33. DuPont HL, Jiang ZD, Okhuysen PC, et al. A randomized, double-blind, placebo-controlled trial of rifaximin to prevent travelers' diarrhea. Ann Intern Med. 2005;142:805-812.

34. DuPont HL, Jiang ZD. Influence of rifaximin treatment on the susceptibility of intestinal Gram-negative flora and Enterococci. Clin Microbiol Infect. 2004;10:1009-1011.

35. Finegold SM, Molitoris D, Vaisanen ML. Study of the in vitro activities of rifaximin and comparator agents against 536 anaerobic intestinal bacteria from the perspective of potential utility in pathology involving bowel flora. Antimicrob Agents Chemother. 2009;53:281-286.

36. Sakata H, Fujita K, Yoshioka H. The effect of antimicrobial agents on fecal flora of children. Antimicrob Agents Chemother. 1986;29:225-229.

37. Samonis G, Gikas A, Anaissie EJ, et al. Prospective evaluation of effects of broad-spectrum antibiotics on gastrointestinal yeast colonization of humans. Antimicrob Agents Chemother. 1993;37:51-53.

38. Clayton EM, Rea MC, Shanahan F, et al. Carriage of Clostridium difficile in outpatients with irritable bowel syndrome. J Med Microbiol. 2012;61:1290-1294.

39. Rajilic-Stojanovic M, Smidt H, de Vos WM. Diversity of the human gastrointestinal tract microbiota revisited. Environ Microbiol. 2007;9:2125-2136.

40. Carroll IM, Ringel-Kulka T, Keku TO, et al. Molecular analysis of the luminal- and mucosal-associated intestinal microbiota in diarrhea-predominant irritable bowel syndrome. Am J Physiol Gastrointest Liver Physiol. 2011;301:G799-G807. 